\title{
Opioid Replacement In Pregnant Mothers With Opioid Use Disorder and Fetal Neurodevelopment: A Review
}

\author{
Caragh Dallas Miller ${ }^{1}$, Elizabeth Nizalik ${ }^{1,2}$, David Grynspan ${ }^{1,2}$ \\ ${ }^{1}$ Faculty of Medicine, University of Ottawa \\ 2 Department of Pathology and Laboratory Medicine, CHEO
}

\section{ABSTRACT}

This paper reviews the published literature regarding neurodevelopmental outcomes in neonates following in utero exposure to opioids. We have summarized available clinical and experimental data. Overall, clinical data is limited and equivocal with most studies showing no significant neurodevelopmental impairments in infants and children exposed to opioids in utero. Various outcome measures assessed language, communication, cognitive, psychomotor, and behavioural outcomes. The equivocality of the data may be a result of the complexity of the cohort and the inability to disentangle the effect of the opioids from the multiple comorbidities. Results from experimental data show that all opioids cross the placental barrier. Mouse studies show biochemical and neurophysiological changes, leading to long-term effects on learning and memory. Some data also suggests that epigenetic and imprinting changes in the central nervous system of mice may lead to multigenerational effects of opioid exposure. Ultimately, the benefits of opioid replacement therapy outweigh its risks, but it should be done in the context of a broader biopsychosocial risk reduction approach. Promoting mother-child bonding and care through skin-to-skin contact, rooming-in, and breastfeeding can reduce severity of neonatal abstinence syndrome and improve outcomes. This cohort of women and children requires advocacy for comprehensive multidisciplinary care.

\section{RÉSUMÉ}

Cet article passe en revue la littérature publiée concernant les résultats neurodéveloppementaux chez les nouveau-nés après exposition in utero aux opioïdes. Nous avons résumé les données cliniques et expérimentales disponibles. Dans l'ensemble, les données cliniques sont limitées et équivoques, avec la plupart des études ne montrant aucune déficience neurodéveloppementale significative chez les nourrissons et les enfants exposés aux opioïdes in utero. Diverses mesures de résultats ont évalué les résultats linguistiques, de communication, cognitifs, psychomoteurs et comportementaux. L'équivocité des données peut être le résultat de la complexité de la cohorte et de l'incapacité à démêler l'effet des opiö̈des des multiples comorbidités. Les résultats des données expérimentales montrent que tous les opiö̈des traversent la barrière placentaire. Les études sur la souris montrent des changements biochimiques et neurophysiologiques, conduisant à des effets à long terme sur l'apprentissage et la mémoire. Certaines données suggèrent également que les changements épigénétiques et d'empreinte dans le système nerveux central des souris peuvent conduire à des effets multigénérationnels de l'exposition aux opiö̈des. En fin de compte, les avantages de la thérapie de substitution aux opioïdes l'emportent sur ses risques, mais cela devrait être fait dans le contexte d'une approche plus large de réduction des risques biopsychosociaux. La promotion des liens et des soins entre la mère et l'enfant par le contact peau à peau, l'accoutumance et l'allaitement peut réduire la gravité du syndrome d'abstinence néonatale et améliorer les résultats. Cette cohorte de femmes et d'enfants a besoin de plaidoyer pour des soins multidisciplinaires complets.

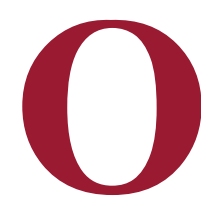

pioid use disorder has become increasingly prevalent worldwide and thus is a topic of public concern [1]. Concomitantly, this is resulting in increased rates of opioid use in pregnancy [2]. The number of infants born to opioid dependent women in Ontario has increased from 46 to almost 800 between 2002 and 2014 [2]. The Society of Obstetrics and Gynaecology of Canada (SOGC) recommends opioid replacement therapy in pregnancy to avoid withdrawal and illicit substance abuse $[3,4]$. The SOGC cites benefits of opioid replacement during pregnancy such as increased birth weight, longer gestation, improved prenatal care, more infants being discharged in the care of their mothers, and decreased complications [3,4].

Opioid replacement can be carried out with full agonist therapy (methadone), partial agonist therapy (buprenorphine), or combined partial agonist and antagonist therapy (buprenorphine plus naloxone) $[3,4]$. In longitudinal studies looking at develop-

Keywords: Opioid use disorder; Opioid replacement; Methadone; Buprenorphine; Neurodevelopment; Outcome studies 
mental outcomes, it is difficult to separate effects of opioid exposure from comorbidities existing in this population including poverty, malnutrition, and co-infection (such as with Hepatitis $B$ and $C$ and HIV) $[3,5]$. Despite the potential effects of opioid replacement on fetal neurodevelopment, the risk of the mother experiencing withdrawal or relapse of illicit drug use when replacement is not provided carries greater morbidity; thus, replacement is recommended $[3,4]$.

Infants exposed to opioids in utero either through illicit use or medical opioid replacement can experience withdrawal symptoms. The manifestation of these withdrawal symptoms in the neonate is referred to as neonatal abstinence syndrome (NAS) [6]. The symptoms of NAS include features of hyperactivity of the central and autonomic nervous system [6]. Hyperactivity of the central nervous system results in symptoms such as jitteriness and tremors. Hyperactivity of the autonomic nervous system results in symptoms such as sweating and mottling of the skin and enteric symptoms such as vomiting and diarrhea. NAS severity is graded with a validated clinical tool, called the Modified Finnegan Scoring System and is managed by pharmacological treatment with oral morphine or methadone [6].

Here, we will review literature on neurodevelopmental outcomes of neonates with in utero exposure to opioids. We note that neonatal abstinence syndrome can also affect fetal neurodevelopment. We will focus on the effects of opioids themselves on the developing brain. We will first review the clinical data (outcome studies) of in utero opioid exposure. We will then discuss the limitations of such studies. Next, we will review existing basic scientific data and summarize what this data suggests in terms of potential (perhaps more subtle) consequences that we should be mindful of.

\section{CLINICAL DATA}

Overall, the majority of data that is older than a decade suggests that there is no significant long-term neurodevelopmental effect of in utero opioid exposure [4]. Recent data is slightly more equivocal, with various levels of deficit being documented in opioid exposed cohorts on general developmental scales (such as the Bayley Scale of Infant Development), motor delays, and changes in Electroencephalographic (EEG) responses to auditory stimulation. These changes were seen in infancy and in some studies, they persisted into toddlerhood [7]. In contrast, one large epidemiological study showed that language development and communication skills were not affected in 3-year- old children who were exposed to analgesic opioid in pregnancy [8]. A meta-analysis published in 2014 aggregated five case-controlled observational studies that compared opioid exposed to non-exposed children, using various quantitative psychometric tests [9]. All five studies involved populations living in urban, low socioeconomic communities. For the sake of the meta-analysis, the outcomes of the tests were grouped into one of three domains: cognitive, psychomotor and behavioural. The meta-analysis found no statistically significant impairment in cognitive, psychomotor, or observed behavioural outcomes in infants and pre-school children exposed to opioids in utero. However, there was a trend to impaired outcomes in all domains [9].

Another group of investigators attempted to examine correlates of neurodevelopment measured in utero $[10,11]$. This was accomplished by assessing the relationship between fetal movement and the integration of movement with cardiac regulation, as measured by Doppler. The authors found that towards the end of gestation, there were reductions in intrauterine fetal movement in fetuses of mothers receiving buprenorphine. They also found a reduction in the coupling of cardiac activity with fetal movement at peak buprenorphine levels ( 2.5 hours following the daily dose) when compared to trough buprenorphine levels (measurements taken immediately prior to the daily dose) $[10,11]$. The authors consider the evolving ability of a developing fetus to match cardiac rate to activity level as a form of neurodevelopment and thus question whether opioids could impact neurodevelopment more broadly.

\section{LIMITATIONS}

Mothers and neonates with opioid exposure in pregnancy have multiple co-morbidities and are therefore very complex. These comorbidities include food insecurity, lack of access to care, additional drug and alcohol dependencies, mental health conditions, exposure to trauma, victimization and violence, malnutrition, and infection. This makes it extremely difficult to disentangle where the risk to neurodevelopment lies [7]. Authors have suggested that sociodemographic factors and effects of the care-taking environment may be more important determinants of developmental outcomes than the biological effects of opioid exposure. These authors have emphasized the need for interventions that support the parent and enrich the child's environment $[5,12,13]$. 
Another limitation of clinical studies is that opioid doses vary widely, which makes it difficult to generalize a given study. Accurate quantification of illegal opioid use is very difficult, and in studies of patients receiving medical replacement, it is difficult to account for potential illicit use during the study interval.

\section{EXPERIMENTAL DATA}

Evidence that opioids cross the placenta

All opioid replacement regimens are known to cross the placenta. Malek et al. reviewed the pharmacology of the interaction of opioids with the placenta [14]. The studies they reviewed included analyses of metabolites within maternal blood and umbilical cords (fetal blood) of mothers exposed to opioids, as well as experiments employing in vitro perfused placental explants. All methodologies suggested that opioids, including morphine, and synthetic opioids (such as fentanyl) can cross the placenta [14].

Mouse data of anatomical CNS variations in mice exposed antenatally to opioids

One elegant and carefully designed experiment demonstrated that mice exposed to morphine prenatally showed early postnatal biochemical and physiological changes, which correlated with changes in learning and memory at older ages [15]. The authors compared total protein and protein phosphorylation levels between the hippocampus of mice that had been exposed to morphine in utero with unexposed controls. They found evidence of altered expression of markers known to be associated with signaling through the N-methyl-D-aspartate receptor (NMDAR), which plays an important role in learning, memory, and development. This included reduced level of a protein called PSD-95, which complexes with the NMDAR, and mediates signaling as well as decreased phosphorylation of the CAMP responsive element binding protein (CREB) at serine 133. CREB is an NMDA activated transcription factor involved in learning and memory. The change in PSD-95 levels was seen at day 14 of life but normalized at later time points [15].

The results also showed that neurophysiological changes were taking place in those mice exposed to morphine in utero [15]. Hippocampal neurons of these mice showed decreased longterm depression of excitatory post-synaptic potentials following a stimulatory voltage pulse, thus showing reduction in a form of synaptic plasticity - a cellular correlate of learning. This study also found persistent long-standing changes in memory and learning. Mice exposed to morphine in utero had impaired performance on the water maze task, which is a classical test of spatial learning and memory. This effect was only seen in the first 2 days of testing and on consecutive days the mice did catch up to their respective controls [15].

Another study showed region-specific changes in the quantities of a specific class of neuron (defined by its expression of the calcium binding protein Calbindin D28-k) in the offspring of mice exposed to morphine throughout gestation [16]. The study looked at expression of Calbindin D-28k in the cingulate cortex, parietal cortex, and hippocampus of opioid exposed versus unexposed neonatal mice. The study found that in certain cortical layers, expression was increased whereas in others it was decreased by opioid exposure. These changes were not seen when adult mice were exposed to opioids. This suggests that immature neurons in the mouse brain have specific sensitivities to opioid exposure during development, which could theoretically affect learning, behaviour, or mood later in life. There is also evidence that maternal exposure to morphine prior to conception, even if there is no fetal exposure in the pregnancy itself, could have biological effects on the fetus. One study showed that when morphine was administered to female rats for five weeks, and then discontinued 4 weeks prior to mating, the offspring showed increased levels of hippocampal tumor necrosis factor alpha (TNFa) and decreased levels of the S100B protein [17]. Hippocampal TNFa has been shown in previous studies to alter memory performance in rats with chronic morphine exposure [18]. S100B protein is mainly expressed by astrocytes, a glial cell, and plays a role in neuro-inflammation and response to CNS damage.

Epigenetic and imprinting changes in the CNS lead to longterm consequences

Human studies have established that epigenetic changes in the promoter of the mu opioid receptor (OPRM1), which is the main site of action of opioids, exist in adults with opioid use disorder $[19,20]$. This was demonstrated by showing a higher frequency of methylation of certain cytosine residues within the OPMR1 promoter, part of $\mathrm{CpG}$ islands, which are areas on the DNA subjected to epigenetic control by methylation. This epigenetic change has been postulated as a potential mechanism contributing to tolerance and addiction. A group of investigators showed similar higher levels of methylation on the OPMR1 promoter in the saliva and blood of infants with neonatal abstinence syndrome. However, the study could not determine whether the epigenetic change was a pre-existing risk factor or 
a change caused by the opioid exposure itself [21].

Animal experiments have suggested that exposure to morphine can cause multigenerational inherited effects. Byrnes et al. showed that when adolescent female mice were exposed to morphine prior to conception, the first generation (F1) and even second generation (F2) offspring showed behavioral and endocrine changes and accompanying molecular changes within the nucleus accumbens [22]. The nucleus accumbens is a dopaminergic system in the basal forebrain that is central to the reward circuit and is integrally implicated in addiction neuroscience. The authors used the locomotor sensitization assay, which is an established behavioural assay wherein animals that are chronically exposed to addictive drugs such as cocaine show hyperactivity following an acute challenge. The study showed that first and second generation progeny of female mice that were given morphine prior to conception show diminished locomotor sensitization in response to a dopamine agonist, quinprole, This was accompanied by reduced corticosteroid release which is mediated through the nucleus accumbens. They also found upregulation of mRNA expression of the kappa opioid receptor and the D2 dopamine receptor in the nucleus accumbens of the F1 and F2 female progeny. This heritability suggests an epigenetic mechanism whereby changes in genomic regulation (such as chromatin methylation or histone modification) are transmitted across generations [22].

As a caveat, we note that extrapolating such results from mouse to human is controversial. For example, the behavioural effects of epigenetic changes are likely to be much stronger in mice than humans, as behaviour in humans is, in general, less deterministic.

\section{CLINICAL APPLICATIONS}

Opioid replacement during pregnancy is currently indicated as part of a realistic risk reduction approach. Uniformly, published consensus statements and practice-guidelines state that the potential risk of in utero opioid replacement is strongly outweighed by its benefits $[3,4,6]$. These benefits include improved prenatal care, longer gestation, and reduced incidences of fetal demise, placental abruption and fetal passage of meconium $[3,4]$. In addition, untreated opioid use disorder is associated with high-risk activities such as prostitution, which expose women to violence, STIs and potential legal consequences [3]. Opioid replacement has also been shown to increase the rate of newborns remaining in the care of their mothers [4].
Long term follow-up and careful studies of language cognition and communication are required. This will help optimize management to ensure the best possible outcome. A potential, but not well-studied, alternative to opioid replacement is supervised withdrawal. This approach is not currently supported because it is associated with high relapse rates (to illicit opioid use) and poorer overall outcomes [3]. A recent study in Northern Ontario at the Meno Ya Win Health Centre in Sioux Lookout demonstrated that narcotic tapering with long-acting morphine preparations can be safely conducted in pregnancy and is associated with significantly lower incidences of NAS [23]. More studies are needed to assess whether supervised withdrawal may have a role, for example, in remote communities where there is no access to a methadone replacement program and where obtaining funding and supply for buprenorphine may be challenging.

As opioid replacement is a harm reduction strategy, it should be part of a comprehensive approach that provides supportive care to the mother, child, and family in order to optimize outcomes. Immediately following birth, skin-to-skin contact is protective against NAS [24]. Enabling a mother to room-in with her newborn while the newborn is getting advanced care or monitoring has also been shown to lower the incidence of NAS $[24,25]$. Providing maternal support to improve rates and duration of breastfeeding is also important, because evidence shows that breastfeeding not only decreases severity of NAS but also reduces maternal stress and enhances maternal confidence and mother-child bonding [6]. Ongoing medical and social support assistance to the family should include chemical dependency and relapse prevention programs [6], nutritional and financial support [26], and screening and support for cooccurring mental health conditions, trauma, and victimization [3]. Preventive interventions that provide enriched environments and high-quality care for infants and children are beneficial to neurodevelopmental outcome [12].

\section{CONCLUSION}

Clinical literature strongly supports opioid replacement in pregnancy for mothers with opioid use disorder and this approach is recommended by most professional guidelines. Opioid replacement prevents withdrawal and relapse and increases participation in antenatal care. Clinical experience and the studies conducted show that the benefits of opioid replacement outweigh its risks and that there are no major short-term developmental risks to the neonate. Careful epidemiological and ex- 
perimental data, however, suggests that there could be subtler neurodevelopmental consequences that merit further study. Opioid replacement is part of a risk reduction strategy and the child's longer-term outcome can address social, psychological and physical injustices and comorbidities experienced by this vulnerable and precious population.

\section{REFERENCES}

1. United Nations Office on Drug and Crime. World drug report. United Nations publication. 2016.

2. Brogly SB, Turner S, Lajkosz K, et al. Infants Born to Opioid-Dependent Women in Ontario. J Obstet Gynaecol Can. 2017;39(3):157-65.

3. Committee on Obstetric Practice. Committee opinion No. 711: Opioid use and opioid use disorder in pregnancy. Obstet Gynecol. 2017;130(2):e81-94.

4. Wong S, Ordean A, Kahan M, et al. SOGC clinical practice guidelines: Substance use in pregnancy. Int J Gynecol Obstet. 2011;114(2):190-202.

5. Kaltenbach K and Finnegan LP. Perinatal and developmental outcome of infants exposed to methadone in-utero. Neurotoxicol Teratol. 1987;9(4):3113.

6. Reddy UM, Davis JM, Ren Z, Greene MF. Opioid Use in Pregnancy, Neonatal Abstinence Syndrome, and Childhood Outcomes. Obstet Gynecol. 2017;130(1):10-28.

7. Logan B, Brown M, Hayes M. Neonatal abstinence syndrome: treatment and pediatric outcomes. Clin Obstet Gynecol. 2013;56(1):186-92.

8. Skovlund E, Handal M, Selmer R, Brandlistuen RE, Skurtveit S. Language competence and communication skills in 3-year-old children after prenatal exposure to analgesic opioids. Pharmacoepidemiol Drug Saf. 2017:26(6);625-34.

9. Baldacchino A, Arbuckle K, Petrie DJ, McCowan C. Neurobehavioral consequences of chronic intrauterine opioid exposure in infants and preschool children: a systematic review and meta-analysis. BMC Psychiatry. 2014:14:104.

10. Jansson LM, Dipietro JA, Velez $M$, et al. Fetal neurobehavioral effects of exposure to methadone or buprenorphine. Neurotoxicol Teratol. 2011;33(2):240-3.

11. Jansson LM, Velez M, McConnell K , et al. Maternal buprenorphine treatment and fetal neurobehavioral development. Am J Obstet Gynecol. 2017;216(5):529.e1-529.e8.

12. Hans SL. Developmental Consequences of Prenatal Exposure to Methadone. Ann N Y Acad Sci. 1989;562:195-207.

13. Messinger DS, Bauer CR, Das A, et al. The maternal lifestyle study: cognitive, motor, and behavioural outcomes of cocaine-exposed and opiate-exposed infants through three years of age. Pediatrics. 2004;113(6):1677-85.

14. Malek A, Mattison DR. Drugs and medicines in pregnancy: the placental disposition of opioids. Curr Pharm Biotechnol. 2011;12(5):797-803.

15. Yang SN, Liu CA, Chung MY, et al. Alterations of post-synaptic density proteins in the hippocampus of rat offspring from the morphine-addicted mother: Beneficial effect of dextromethorphan. Hippocampus. 2006;16(6):521-30.

16. Mithbaokar P, Fiorito F, Della Morte R, Maharajan V, Costagliola A. Chronic maternal morphine alters calbindin D-28k expression pattern in postnatal mouse brain. Synapse. 2016;70(1):15-23.

17. Amri J, Sadegh M, Moulaei N, Palizvan MR. Transgenerational modification of hippocampus TNF- $\alpha$ and S100B levels in the offspring of rats chronically exposed to morphine during adolescence. Am J Drug Alcohol Abuse. 2017:1-8.

18. Pan J, He L, Li X, et al. Activating autophagy in hippocampal cells alleviates the morphine-induced memory impairment. Mol Neurobiol. 2016:54(3):1710-24.

19. Goldman D, Oroszi G, Ducci F. The genetics of addictions: uncovering the genes. Nat Rev Genet. 2005;6(7):521-32.

20. Levran O, Yuferov V, Kreek MJ. The genetics of the opioid system and spe- cific drug addictions. Hum Genet. 2012;131(6):823-42.

21. Wachman EM, Hayes MJ, Lester BM, et al. Epigenetic variations in the mu-opioid receptor gene in infants with neonatal abstinence syndrome. 2015;165(3):472-8

22. Byrnes JJ, Johnson NL, Carini LM, Byrnes EM. Multigenerational effects of adolescent morphine exposure on dopamine D2 receptor function. Psychopharmacology (Berl). 2013;227(2):263-72.

23. Dooley R, Dooley J, Antone I, et al. Narcotic tapering in pregnancy using long-acting morphine: An 18-month prospective cohort study in northwestern Ontario. Can Fam Physician. 2015;61(2):e88-95.

24. Ordean A, Kahan M, Graves L, Abrahams R, Kim T. Obstetrical and Neonatal Outcomes of Methadone-Maintained Pregnant Women: A Canadian Multisite Cohort Study. J Obstet Gynaecol Canada. 2015;37(3):252-7.

25. Newman A, Davies GA, Dow K, et al. Rooming-in care for infants of opioiddependent mothers Implementation and evaluation at a tertiary care hospital. Can Fam Physician. 2015;61(12):555-61.

26. Goodman D. Improving Access to Maternity Care for Women with Opioid Use Disorders: Colocation of Midwifery Services at an Addiction Treatment Program. J Midwifery Womens Health. 2015;60(6):706-12. 